lated well with the random bladder scans performed on other infants, where only $33 \%$ had urine detected in their bladder at the time of initial scanning. The technique of bladder scanning for urine content can be taught to resident medical staff in less than 10 minutes.

With the increasing availability of ultrasound machines on neonatal wards, paediatric wards, and even in the community, bladder aspiration will present less of a problem to medical staff and result in less pain to babies.

\begin{abstract}
References
' Normand A, Smellie J. The child with urinary tract infections. Medicine 1977;28:1588-9.

${ }^{2}$ Braude H, Forfar JO, Gould JC, McLeod JW. Cell and bacterial counts in the urine of normal infants and children. $\mathrm{Br}$ Med $J$ 1967;29:297-9.

${ }^{3}$ Nelson JD, Peters PC. Suprapubic aspiration of urine in premature and term infants. Pediatrics 1965;36:132-3.
\end{abstract}

Correspondence to Dr C O'Callaghan, Department of Child Health, University Hospital, Nottingham, England.

Received 23 April 1987

\title{
Early congenital syphilis and severe haematological disturbance
}

\author{
M C G STEVENS, ${ }^{*}$ P J DARBYSHIRE, ${ }^{*}$ AND S M BROWN $\dagger$ \\ ${ }^{*}$ Departments of Oncology and Haematology, Children's Hospital, Birmingham, and +Department of \\ Paediatrics, Walsgrave Hospital, Coventry
}

\begin{abstract}
SUMmaRY The early clinical signs and symptoms of congenital syphilis are diverse but, if undiagnosed, signs of the disease may subside until the late stigmata appear. We report a case that illustrates that the haematological signs and symptoms may be so severe as to mimic a diagnosis of leukaemia or disseminated malignant disease.
\end{abstract}

Early congenital syphilis is rarely diagnosed in the United Kingdom; an average of 12 cases a year were reported between 1973 and $1982 .{ }^{1}$ Clinical signs are usually absent at birth and during the first few weeks of life. The earliest findings may be non-specific and subsequent symptoms and signs are diverse; if untreated they may subside until the stigmata of late congenital syphilis appear. The chief pitfall in the diagnosis of early infection is paediatricians' failure to include it among the differential diagnoses of a large number of illnesses in infancy. We report a child with early congenital syphilis who presented with such profound haematological disturbance that leukaemia or disseminated malignant disease were considered to be the most likely diagnoses.

\section{Case report}

An 8 week old baby girl presented with a one week history of rash, irritability, and increasing pallor. She had been born at 36 weeks' gestation, weighing $2400 \mathrm{~g}$, after a pregnancy complicated only by uncertain dates. Serological screening at about 22 weeks' gestation showed a negative reaction for syphilis using the Venereal Diseases Research Laboratory (VDRL) and Treponema pallidum haemagglutination (TPHA) tests. She had mild jaundice which was treated with phototherapy for 24 hours and subsequently resolved. On examination she was feverish with extreme pallor and a widespread rash characterised by circular, erythematous (almost papular) lesions, and some superficial scaling. There were a few bruises over the trunk and the liver edge was palpable $8 \mathrm{~cm}$, and the spleen tip 6 $\mathrm{cm}$, below the costal margins. Examination of the fundi, mucosal surfaces, perianal region, and genitalia yielded normal results.

Initial haematological investigations showed a haemoglobin concentration of $2.9 \mathrm{~g} / \mathrm{dl}$, a platelet count of $<10 \times 10^{9} / 1$, a white cell count of $22.4 \times 10^{9} / 1$ (neutrophils $11 \%$, lymphocytes $61 \%$, monocytes $4 \%$, metamyelocytes $3 \%$, myelocytes $1 \%$, blast cells $20 \%$ ), and the ratio of nucleated red cells to white cells was 2:100. Examination of the peripheral blood smear confirmed the presence of immature 'blastlike' cells. Coagulation screen yielded normal and direct antiglobulin test negative results. Despite the severity of the thrombocytopenia and poor initial response to transfusion of platelets there was no obvious evidence of bleeding. Liver function tests were abnormal with aspartate aminotransferase activity of $66 \mathrm{IU} / 1$ (normal $<50$ ); alanine aminotransferase activity of $60 \mathrm{IU} / 1$ (normal $<40$ ); alkaline phosphatase activity of 1147 IU/l (normal for age); bilirubin concentration of $44 \mu \mathrm{mol} / \mathrm{l}$ (normal <17); direct bilirubin concentration of $<5$ (normal); total protein concentration of $49 \mathrm{~g} / \mathrm{l}$ (normal 55-75); and albumin concentration of $15 \mathrm{~g} / 1$ (normal 30-50). 
Chest $x$-ray picture was normal, but a skeletal survey showed periosteal reaction along the shafts of the long bones with some metaphyseal irregularity.

Bone marrow aspirates were obtained from the anterior tibia and both posterior iliac crests. The samples were consistently hypocellular but showed a population of immature cells $(25 \%)$, with morphological features suggesting monocytoid differentiation. There were, in addition, appreciable numbers of large histiocytic cells $(17 \%)$, indicating erythrophagocytosis. Cytochemical stains using periodic acid Schiff, Sudan black, and alphanapthyl esterase were negative. A few blast cells were faintly positive to acid phosphatase. Surface marker studies with a panel of monoclonal antibodies showed a broad response (My9 40\%, Mol 49\%, SmIg 30\%, I $30 \%$, My $725 \%$, J5 <5\%, and Tdt negative).

Disseminated malignant disease seemed to be the most likely diagnosis, with malignant histiocytic disease, acute monocytic leukaemia, or histiocytosis $\mathrm{X}$ as the most favoured alternatives. The possibility
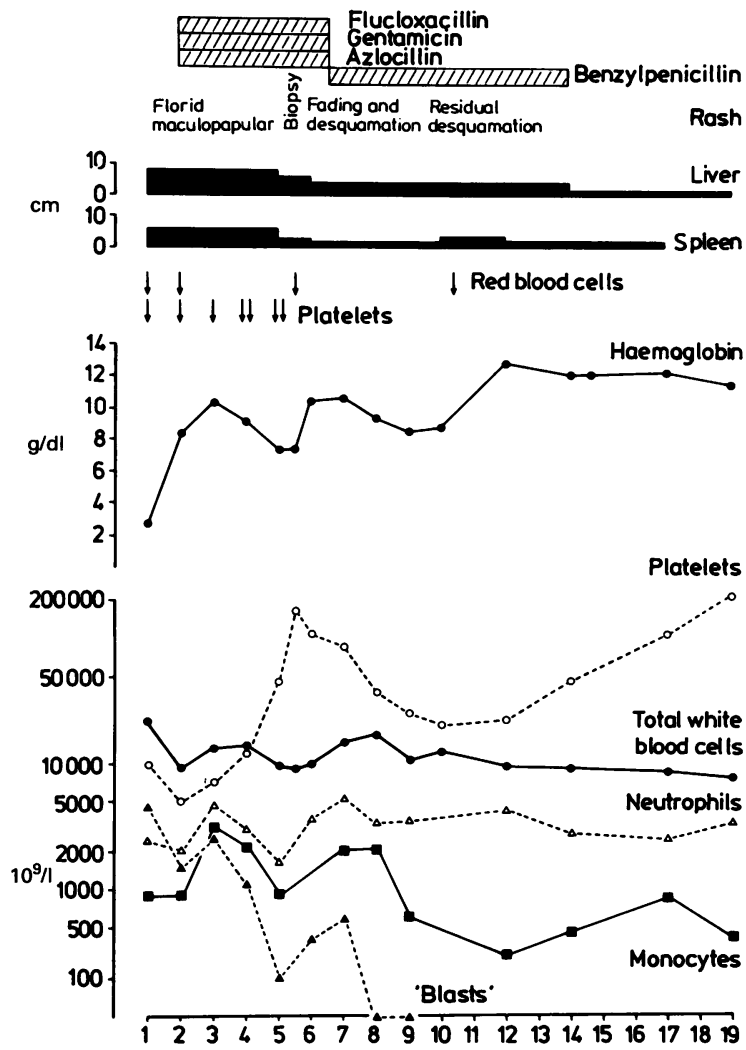

Figure Clinical, haematological, and therapeutic data from time of admission. of congenital infection was considered but thought less likely in view of the severity of the haematological abnormalities. Initial management included transfusions of blood and platelets and, pending results of bacterial cultures and viral serology, treatment with broad spectrum antibiotics (azlocillin, gentamicin, and flucloxacillin). In view of the difficulty in interpreting the haematological results a skin biopsy was performed. It was notable, however, that the skin rash had improved after antibiotic treatment, as had the infant's general condition, with resolution of the fever and regression of the hepatosplenomegaly (figure). On histological examination the skin biopsy specimen showed a histiocytic/lymphocytic infiltration, although the appearances were not diagnostic.

Six days after admission, VDRL and TPHA tests were reported as being positive in samples taken on admission from both mother and baby, suggesting a diagnosis of congenital syphilis. Subsequent results from the reference laboratory confirmed this as follows: baby-VDRL 1:64, TPHA 1:512, and cerebrospinal fluid negative to both; motherVDRL 1:64, and TPHA 1:1024. Antibiotic treatment was changed to benzyl penicillin, the total dose exceeding the WHO guidelines for adequate treatment. $^{2}$ The baby made an uneventful recovery. The rash desquamated and faded, hepatosplenomegaly regressed completely, and the blood count returned to normal after 10 days. The family was referred to their local genitourinary clinic for follow up and the baby remained well and was developing normally one year after diagnosis.

\section{Discussion}

The characteristic clinical changes of early congenital syphilis are widespread and include rash, severe rhinitis, moist lesions at mucocutaneous junctions, painful limbs (often presenting as pseudoparalysis), lymphadenopathy, and hepatosplenomegaly. Jaundice is common, as is hypoproteinaemia which may cause oedema. Of the haematological findings, anaemia commonly occurs; almost $90 \%$ of children with early congenital syphilis diagnosed postnatally were described as anaemic in a recent large review. ${ }^{3}$ The incidence of thrombocytopenia is not clear, although the association of increased platelet turnover (with or without thrombocytopenia) with congenital viral infections, syphilis, and toxoplasmosis is well known. ${ }^{4}$ The severity of the marrow reaction in this child was striking, with prominent immature monocytoid and mature histiocytic cell populations together with a leucoerythroblastic response in the peripheral blood, mimicking disseminated malignant disease. A similar but less 
severe presentation was described in one of nine infants with haematological signs and symptoms of congenital syphilis. ${ }^{5}$

The negative results of the antenatal serological tests imply that the mother was either incubating the infection at that time, or had acquired it later in pregnancy. A similar sequence of events was seen in five of seven women whose infants were later diagnosed as having early congenital syphilis in a survey from the Mersey region, ${ }^{1}$ which raises the question of whether serological screening should be repeated during the third trimester.

The case we report emphasises the serious implications of early congenital syphilis, and shows that the haematological abnormalities may sometimes be severe.

We thank the department of illustration, Birmingham Children's
Hospital, Jacqueline Boaler for preparation of the manuscript, and Dr JC Clay for helpful discussion.

References

${ }^{1}$ Ewing CI, Roberts C, Davidson DC, Arya OP. Early congenital syphilis still occurs. Arch Dis Child 1985;60:1128-33.

2 World Health Organisation. Treponemal infections. Report of a WHO Scientific Group. Geneva, 1982:674.

${ }^{3}$ Hira SK, Bhat GJ, Patel JB, et al. Early congenital syphilis: clinico radiologic features in 202 patients. Sex Transm Dis 1985;12:177-83.

${ }^{4}$ Stockman JA. Hematologic manifestation of systemic diseases. In: Nathan DG, Oski FA, eds. Hematology of infancy and childhood. Philadelpha: WB Saunders, 1981:1339-87.

5 Whitaker JA, Sartain P, Shaheedy M. Hematological aspects of congenital syphilis. J Pediatr 1965;66:629-36.

Correspondence to Dr MCG Stevens, Department of Oncology, Children's Hospital, Ladywood, Birmingham B16 8ET.

Received 26 May 1987

\title{
Post-streptococcal glomerulonephritis in Hong Kong
}

\author{
D T Y LEUNG, R Y M TSENG, S H GO, G L FRENCH, AND C W K LAM
}

Departments of Paediatrics, Microbiology, and Chemical Pathology, Prince of Wales Hospital, The Chinese University of Hong Kong, Shatin, New Territories, and Department of Paediatrics, United Christian Hospital, Kwun Tong, Kowloon

SUMMARY Of 74 paediatric inpatients with acute glomerulonephritis, $58(78 \%)$ had a raised $(>1 / 200)$ antistreptolysin $O$ titre. Serum C3 concentration was low in 73 , but returned to normal within six weeks. Streptococcal infection remains the commonest cause of acute nephritis in children in Hong Kong, possibly due to overcrowded living conditions.

Over the past 20 years the incidence of acute glomerulonephritis in developed countries has fallen considerably, and the aetiological role of streptococcal infection seems also to have waned. Meadow in 1975 found that only. one third of 43 children in Leeds with acute nephritis had had a recent streptococcal infection. ${ }^{1}$

The pattern of illness in children in Hong Kong is similar to that in other developed parts of the world, ${ }^{2}$ yet it was thought that acute glomerulonephritis was more common than in Western countries. We therefore set up a prospective study to determine whether recent streptococcal infection remains a major cause of acute glomerulonephritis in these children.

\section{Patients and methods}

From July 1984 to July 1986 we studied all children with acute glomerulonephritis admitted to two regional hospitals, one in Kowloon and the other in the New Territories. Criteria for diagnosis comprised gross or microscopic haematuria and at least two of the following: diastolic blood pressure of $>80$ $\mathrm{mmHg}$, plasma urea concentration $>6.6 \mathrm{mmol} / \mathrm{l}$, oedema or oliguria. ${ }^{1}$ Children with primary renal disorders were not included.

Seventy four children with suspected acute nephritis were studied: 43 boys and 31 girls, aged from 8 months to 15 years. Particular attention was taken of any throat, respiratory tract, or skin infections in the previous four weeks. We also asked if the children had seen a doctor before admission, and if they had received any medication. Throat swabs for culture and blood specimens for estimation of antistreptolysin O (ASO) titre and assay of C3 complement concentration in serum were taken within five days of admission. Throat swabs were placed in Stuart's transport medium before being sent to the laboratory for culture on blood agar in carbon dioxide. ASO titre was assayed using a modified microtechnique with a microtitre plate; a 